\title{
El joven Foucault y la crítica de la razón psicológica: en torno a los orígenes de la Historia de la locura
}

\author{
The young Foucault and the critique of psychological \\ reason: On the origins of Madness and Civilization
}

\author{
ENRIC J. NOVELLA* \\ Centro de Ciencias Humanas y Sociales (CSIC), Madrid
}

Resumen. Partiendo de la amplia repercusión y del lugar germinal que la Historia de la locura en la época clásica (1961) ocupa en el desarrollo del pensamiento de Michel Foucault, y con el objeto de esclarecer sus antecedentes teóricos y su complejo proceso de gestación, este artículo ofrece un análisis de los escritos tempranos del filósofo francés en torno a los problemas de la psiquiatría y la psicología. En primer lugar, se aborda su posición con respecto a las premisas conceptuales de la medicina mental y se señalan las insuficiencias que detectó en las principales corrientes de la psicopatología de la época (fundamentalmente, el evolucionismo, el psicoanálisis y la fenomenología). Posteriormente, se describe su primer intento de interpretar globalmente el fenómeno de la enfermedad mental desde un punto de vista social y cultural a partir de la teoría marxista de la alienación y la reflexología pavloviana. Y, por último, se muestra cómo fueron perfilándose en su producción de aquellos años algunos de los elementos y planteamientos más significativos que, al cabo de un tiempo, cristalizarían y encontrarían una forma acabada en la Historia de la locura.

Palabras clave: Michel Foucault, psicopatología, psicología, psiquiatría, historia de la locura.
ABSTRACT. Taking into account the wide influence and the germinal role of Madness and Civilisation (1961) in the development of Michel Foucault's thought, and in order to clarify its theoretical background and its complex genesis, this article provides an analysis of the early writings of the French philosopher on the problems of psychiatry and psychology. First, I examine his position concerning the conceptual assumptions of mental medicine and the shortcomings he detected in the main currents of psychopathological thought (basically, evolutionism, psychoanalysis, and phenomenology). Afterwards, I describe his first attempt to outline a global interpretation of the phenomenon of mental illness from a social and cultural standpoint resorting to Marxist alienation theory and Pavlovian reflexology. And, finally, I show how some of the most significant elements and insights that, within a short time, would crystallise and find a final form in Madness and Civilisation, were gradually shaped along his production of those years.

Key words: Michel Foucault, psychopathology, psychology, psychiatry, history of madness.

* Investigador contratado doctor (Programa JAE-Doc). Dirección para correspondencia: C/ Albasanz 26-28, 28037 Madrid (España).Correo electrónico: enric.novella@cchs.csic.es 
«En réalité, c'est dans l'histoire seulement que l'on peut découvrir les conditions de possibilité des structures psychologiques»

Michel Foucault (1954)

\section{La Historia de la locura y sus consecuencias}

En mayo de 1961, Michel Foucault presentó en la Sorbona y ante un tribunal presidido por el historiador de la filosofía Henri Gouhier una voluminosa y, en muchos sentidos, innovadora tesis doctoral con el título Locura y sinrazón: Historia de la locura en la época clásica. El trabajo acababa de ser publicado por la editorial académica parisina Plon, y, aunque Foucault se quejó más tarde de una acogida más bien fría (Eribon, 1992, pp. 145-176), alcanzó rápidamente el rango de un clásico. Desde una perspectiva actual puede decirse que resulta prácticamente imposible reseñar la profunda y persistente influencia del libro, y que ésta difícilmente puede sobreestimarse, al menos en el ámbito de la filosofía, la historiografía o la propia psiquiatría ${ }^{1}$.

Desde un punto de vista filosófico, la Historia de la locura fue el primer gran hito en el desarrollo de un pensamiento altamente original e influyente que rebasaba ya los anteriores marcos de referencia teóricos del joven Foucault - particularmente, la fenomenología y el marxismo. Ciertamente, el concepto clave seguía siendo el de experiencia, pero su programa ya no consistía en el esclarecimiento de las condiciones de posibilidad de cualquier experiencia, sino en la dilucidación de los requisitos históricos y estructurales que habían conformado y hecho posible el objeto de un determinado saber - en este caso, la psiquiatría y la psicología - y una determinada experiencia - aquí, la experiencia moderna de la locura. De este modo, Foucault inauguró un acercamiento teórico y metodológico que más tarde explicitó y refinó con los conceptos programáticos de la arqueología y la genealogía, pero cuyo núcleo filosófico quedaba ya perfectamente delineado en esta primera gran obra: los fenómenos - como, en este caso, las enfermedades mentales - debían ser despojados de su aparente naturalidad y evidencia, siendo remitidos sistemáticamente a sus condiciones históricas y estructurales de aparición. Foucault describió retrospectivamente este planteamiento y su punto de partida filosófico como una «ontología histórica» ${ }^{2}$ (del presente, de nosotros mis-

${ }^{1}$ La bibliografía sobre la Historia de la locura y su recepción es, de hecho, inabarcable. Véanse, por ejemplo, los trabajos de Barthes (1961), Mandrou (1962), Derrida (1964), Cranston (1973), Russ (1979), Midelfort (1980), Sedgwick (1981), Stone (1982), Flaherty (1986), Macherey (1986), Roudinesco (1992), Still/Velody (1992), Jones/Porter (1994), Gutting (1994), Gros (1997), Gutting (2005) o los números monográficos de revistas como Évolution psychiatrique (1971) o History of the Human Sciences (1990).

2 Como es sabido, esta denominación procede de un texto tardío sobre el ensayo de Kant ¿Qué es Ilustración?, en el que Foucault contrapone a la tradición de la filosofía como «analítica de la verdad» la concepción alternativa de una «ontología histórica»: «Esta otra tradición 
mos) centrada, alternativamente, en los tres ejes principales de la experiencia humana (saber, poder y subjetividad), pero cuyo primer eslabón fue, en todo caso, la Historia de la locura ${ }^{3}$.

Como es sabido, el libro ha recibido con los años una serie de críticas, en buena medida justificadas, a causa de la dudosa validez empírica de algunas de sus afirmaciones ${ }^{4}$. Pero, aun así, es imposible obviar su profunda influencia en el campo de la historiografía. La Historia de la locura fue concebida como una primera contribución a la determinación de los límites de la experiencia y las escisiones constitutivas del mundo moderno. Esta estrategia de análisis del presente o de diagnóstico de la Modernidad por medio del recurso a materiales históricos escogidos — que R. Castel (1994) ha definido posteriormente como «problematización»— ha sido acogida críticamente por muchos historiadores a causa de su manejo a menudo selectivo de los hechos, pero también es cierto que ha desplazado la atención historiográfica hacia nuevos temas y ha estimulado decisivamente el desarrollo de áreas de investigación como la historia cultural, la historia de las instituciones o la historia de la ciencia ${ }^{5}$.

Asimismo, la Historia de la locura contribuyó singularmente a que la psiquiatría encontrara una nueva relación con su historia en un período decisivo como fueron las décadas de los sesenta y setenta del siglo pasado. La gran eclosión de críticas a la institución psiquiátrica y los ambiciosos proyectos de reforma asistencial esbozados entonces debieron buena parte de su fuerza y su dinamismo a esta nueva conciencia histórica en relación con los problemas de la praxis psiquiátrica (Blasius, 1986; Huertas, 2006). De hecho, la provocativa visión foucaultiana de la psiquiatría como una estructura de saber-poder inseparable de su función disciplinaria promovió no sólo una nueva forma de acercarse a su historia ${ }^{6}$, sino también una nueva actitud por parte de mu-

crítica se plantea la cuestión: ¿En qué consiste nuestra actualidad? ¿Cuál es el campo actual de experiencias posibles?» (Foucault, 1994e, p. 687). Y en un importante texto del mismo período prosigue: «La tarea de la filosofía como análisis crítico de nuestro mundo es algo que es cada vez más importante. Quizá el más importante de todos los problemas filosóficos es el problema del presente, de lo que somos en este mismo momento» (Foucault, 1983, p. 216).

3 «Con respecto a su obra subsiguiente, la Historia de la locura tiene un rol germinal y crucial. [...] Sienta los métodos básicos, los problemas y los valores que informan todo lo que escribió después» (Gutting, 1989, pp. 109-110). En este sentido, véase también Deleuze (1986), pp. 75-158, o Sarasin (2005), pp. 17-22.

4 Entre éstas cabe mencionar, en primer lugar, la suposición de que la experiencia de la locura durante el Renacimiento no se hallaba tan marcada por la exclusión y el rechazo como en épocas posteriores, $y$, en segundo lugar, las dimensiones del supuesto «gran encierro» de locos y dementes que Foucault creyó constatar en los siglos XVII y XVIII. Véase una síntesis en Gutting (1989), pp. 49-56.

5 Sobre la influencia de la obra de Foucault en la historiografía posterior pueden consultarse los trabajos de Poster (1982), Megill (1987), Veyne (1992), Goldstein (1994), Lloyd/Thacker (1997) y Flynn (2005).

6 Entre los muchos estudios histórico-psiquiátricos que retomaron y ampliaron los planteamientos críticos de Foucault cabe mencionar los de Dörner (1969), Rothman (1971), Scull 
chos profesionales de la salud mental, para quienes su trabajo y sus hospitales dejaron de ser sinónimos espontáneos de «liberalismo», «humanidad»o «progreso». En consecuencia, y aun cuando algunos de sus planteamientos hayan podido resultar abiertamente cuestionables — como esa «escisión originaria y fatal» entre la razón y la locura que Foucault sitúa en el advenimiento de la Modernidad y personaliza en Descartes ${ }^{7}$, o su idea de la locura como portadora de un lenguaje perdido, silenciado y, en principio, inaccesible para el hombre moderno-, la Historia de la locura permanece como un texto esencial para entender los grandes debates que han jalonado el desarrollo de la psiquiatría actual.

Teniendo en cuenta su amplia recepción y su perdurable influencia, es un tanto sorprendente que la trayectoria de Foucault anterior a la Historia de la locura, que eventualmente podría suministrar algunas claves importantes para el esclarecimiento de la génesis intelectual de su obra y de su evolución teórica posterior, haya recibido, en líneas generales, poca atención ${ }^{8}$. Como es sabido, Foucault completó a principios de los años cincuenta una formación teórica y práctica como psicólogo, e incluso llegó a plantearse la posibilidad de estudiar medicina para dedicarse a la psiquiatría (Eribon, 1992, pp. 70-80; Miller, 1993, pp. 45-92; Macey, 1994, pp. 27-61). Fruto de esa experiencia y de sus primeras inquietudes teóricas, el joven filósofo publicó una serie de trabajos — que incluyen dos capítulos para obras colectivas ( «La psicología desde 1850 a 1950» y «La investigación científica y la psicología», ambos publicados en 1957), un libro introductorio para una colección divulgativa (Enfermedad mental y personalidad, 1954) y un extenso prólogo a la traducción francesa del ensayo de L. Binswanger El sueño y la existencia (también aparecido originalmente en 1954) ${ }^{9}$ - en los que se ocupaba ya intensamente de los problemas de la psiquiatría y la psicología. Ciertamente, la lectura de estos trabajos transmite la sensación de que Foucault «experimentó entonces con diversos conceptos y enfoques teóricos, pero sin comprometerse en exce-

(1979) y Castel (1980). Engstrom/Weber/Hoff (1999) ofrecen un balance de conjunto de la amplia recepción y repercusión de la Historia de la locura en la historiografía psiquiátrica posterior.

7 En esta dirección fue justamente la conocida crítica de Derrida (1964), que ha sido asumida en términos muy similares por otros autores. Véase Flaherty (1986).

8 Una buena muestra de ello es el hecho de que, salvo algunos trabajos aislados como los de Chebili (2005) o Moreno Pestaña (2006), ni siquiera las introducciones más conocidas a la obra de Foucault suelen contener más que algunas consideraciones generales sobre este período. G. Gutting es incluso de la opinión de que existe una llamativa «falta de constancia de las importantes e interesantes ideas del joven Foucault con respecto a la naturaleza y la explicación de la enfermedad mental» (Gutting, 1989, p. 56).

9 Respectivamente, cito estos trabajos por las siguientes ediciones: Foucault 1994a, 1994b, 1984 y 1999. Enfermedad mental y personalidad tuvo una segunda edición en 1962 con el título Enfermedad mental y psicología, a la que me referiré más adelante y que no ha sido traducida al español. Hay que recordar, además, que Foucault realizó en esos años en colaboración con D. Rocher una traducción de Der Gestaltkreis, la conocida obra del fundador de la escuela alemana de medicina antropológica V.v. Weizsäcker. Véase Von Weizsäcker (1958). 
so» (Sarasin, 2005, p. 17). Sin embargo, una lectura atenta de los mismos también permite apreciar que, en realidad, anticipó en ellos muchos planteamientos conocidos de su obra posterior, como es el caso de su crítica de la psicología y la psiquiatría positivistas, de su ambivalente posición frente al psicoanálisis, de su particular recepción de la tradición marxista y fenomenológica o, más específicamente, del proyecto contenido en la Historia de la locura de una «arqueología de la psicología» en el marco de una ontología histórica de las estructuras de experiencia más representativas de la Modernidad.

En lo que sigue, me propongo ofrecer un análisis de esta fase temprana en la obra de Foucault, tratando de reconstruir la travesía intelectual que le llevó sobre la novedosa senda que supuso en su momento la Historia de la locura. Para ello, será necesario, en primer lugar, recordar brevemente su posición inicial con respecto a los problemas planteados por la enfermedad mental y las insuficiencias que detectó en las principales corrientes de la psicopatología de la época (fundamentalmente, el evolucionismo, el psicoanálisis y la fenomenología). Posteriormente, pasaré a describir su primer intento de interpretar globalmente el fenómeno de la enfermedad mental desde un punto de vista social y cultural sirviéndose de la teoría marxista de la alienación y la reflexología pavloviana. Y, por último, intentaré mostrar cómo fueron perfilándose en su producción de aquellos años algunos de los elementos y planteamientos más significativos que, al cabo de un tiempo, cristalizarían y encontrarían una forma acabada en la Historia de la locura.

\section{El joven Foucault y la psicopatología}

Puede decirse sin más dilación que el principal estímulo o punto de partida de las preocupaciones teóricas de Foucault con respecto a la psicología fue su profundo malestar con una disciplina que desde sus inicios como tal se había prescrito un positivismo «que pretende agotar el contenido significativo del hombre con el concepto reductor de homo natura» (Foucault, 1999, p. 66). Esta orientación de la psicología según el modelo de la ciencia natural era para él una clara herencia del pensamiento moderno e ilustrado, y se basaba en dos postulados que describió del siguiente modo: «que la verdad del hombre se agota en su ser natural, y que el camino de todo conocimiento científico debe pasar por la determinación de relaciones cuantitativas, la construcción de hipótesis y la verificación experimental» (Foucault, 1994a, p. 148).

En la crítica de esta psicología caracterizada por «presupuestos naturalistas y el olvido del sentido» (Foucault, 1994b, p. 171), el joven Foucault podía remitirse a una rica tradición que, con el psicoanálisis y la fenomenología a la cabeza, había acometido a lo largo de la primera mitad del siglo XX un severo cuestionamiento de los fundamentos epistemológicos del positivismo psicológico. En particular, las fuentes primordiales que inspiraron la crítica fou- 
caultiana fueron tres. En primer lugar, el desenmascaramiento por parte de M. Heidegger y M. Merleau-Ponty de la filosofía de la conciencia cartesiana y kantiana y su escisión sujeto/objeto como el fundamento último de la visión mecanicista y fragmentaria de la vida psíquica que había conducido a la concepción naturalista de la psicología ${ }^{10}$. En segundo lugar, la insistencia de G. Canguilhem en la historicidad de las objetividades psicológicas y en la difícil aplicación del concepto de normalidad en el ámbito de la psicología ${ }^{11}$. Y, por último, la reivindicación por parte de G. Politzer de una "psicología concreta» centrada en la reconstrucción idiográfica de la historia individual en oposición a cualquier forma de «metapsicología abstracta» ${ }^{12}$.

Análogamente, Foucault cuestionó también de forma precoz las «trampas conceptuales» de la psicopatología clínica y la psiquiatría, rechazando de forma enfática y genérica el concepto mismo de «enfermedad mental». En su opinión, era un error dar «el mismo sentido a las nociones de enfermedad, síntoma y etiología en patología mental y en patología orgánica», pues «la raíz de la patología mental no debe estar en una especulación sobre cierta "metapatología"» que asume que «las perturbaciones orgánicas y las alteraciones de la personalidad [poseen] una causalidad del mismo tipo» (Foucault, 1984, p. 10). A lo largo de las páginas de Enfermedad mental y personalidad, el joven Foucault insiste en que «la patología mental exige métodos de análisis diferentes de los de la patología orgánica, y que sólo mediante un artificio del lenguaje podemos prestarle la misma significación a las "enfermedades del cuerpo" y a las "enfermedades del espíritu"» (Ibid., p. 20). La inadecuación de este «artificio» se deriva, a su juicio, de tres consideraciones fundamentales. En primer lugar, la abstracción de elementos aislados y el análisis de sus vínculos causales, factible en principio para la medicina somática, resulta del todo inapropiada en el campo de la psicología, pues cada elemento de la vida psíquica incluye «el estilo, el modo general, toda la anterioridad y las eventuales implicaciones de una existencia» (Ibid., p. 21). En segundo lugar, la distinción entre lo normal y lo patológico es altamente problemática en el campo de la psiquiatría, pues el concepto de personalidad «sólo permite una apreciación cualitativa que autoriza todas las confusiones» (Ibid., p. 23).

10 Véase Dreyfus (1987). Foucault, además, parece haber estado familiarizado con la conocida obra del psiquiatra alemán E. Straus, Vom Sinn der Sinne (1935), que constituye uno de los análisis más incisivos y profundos de las raíces cartesianas de la psicología positivista. Cf. Foucault (1999), p. 102.

11 Cf. Labreure (2004), pp. 15-17. Canguilhem, de hecho, pronunció a mediados de los años cincuenta una importante conferencia en la que describió los problemas epistemológicos suscitados por la psicología en términos muy similares a los de Foucault: «Con la mayoría de los estudios de psicología se tiene la impresión de que combinan una filosofía sin rigor, una ética sin exigencia y una medicina sin control» (Canguilhem, 1968, p. 366).

12 Politzer, que se convirtió luego en un importante teórico marxista, había publicado en 1928 la Critique des fondements de la psychologie, un texto que Foucault leyó con entusiasmo durante los años de formación en la Ecole Normale Supérieur (Eribon, 1992, p. 56). 
Y, por último, la naturaleza dialéctica de las relaciones del individuo con su medio obliga a la psicopatología a asumir una perspectiva necesariamente ecológica, obliterando la posibilidad de considerar al individuo enfermo de forma aislada. En consecuencia,

«no podemos admitir de lleno ni un paralelismo abstracto ni una unidad masiva entre los fenómenos de la patología mental y los de la orgánica; y es imposible transportar de una a la otra los esquemas de abstracciones, los criterios de normalidad o la definición del individuo enfermo» (Ibid., p. 24).

Una vez descartada la adecuación del modelo médico tradicional para aprehender la singularidad de los fenómenos psicopatológicos, Foucault ofreció un interesante análisis de los logros e insuficiencias de las distintas corrientes de la psicopatología que, en su opinión, habían respetado esta «originalidad radical» de los trastornos mentales. Partiendo de un continuo de menor a mayor concreción y complejidad de sentido, estas corrientes eran el evolucionismo, el psicoanálisis y la fenomenología, cuyas dimensiones psicológicas de referencia se corresponden, respectivamente, con la evolución, la historia individual y la existencia.

Con respecto al evolucionismo, Foucault concede que la recepción del darwinismo por parte de H. Spencer y su transmisión desde el campo de la biología al de la psicología y la sociología supuso en su momento una considerable renovación de las ciencias humanas. Entre otras cosas, el evolucionismo permitió describir simultáneamente la evolución del individuo como un proceso de diferenciación horizontal forjador de diversidad y como un movimiento vertical de integración jerárquica: «así han procedido las especies en el curso de su evolución, así procederán las sociedades en el curso de su historia, así procede el individuo en el curso de su génesis psicológica» (Foucault, 1994a, p. 152). Con la obra de autores como el neurólogo J.H. Jackson, el psicólogo T. Ribot o, más tarde, el psiquiatra H. Ey, el pensamiento evolucionista penetró en el campo de la psicopatología, y desde entonces, señala Foucault, «ya no es posible omitir los aspectos regresivos de la enfermedad; la evolución es, desde entonces, una de las dimensiones por las cuales se tiene acceso al hecho patológico» (Foucault, 1984, p. 33). De todas formas, la perspectiva evolucionista tiene dos serias limitaciones que, a su juicio, la hacen insatisfactoria. Por un lado, tiende a obviar la organización específica de la personalidad mórbida, que

«no es originaria, sino rigurosamente original. [...] Por más profunda que sea la disolución [...] la personalidad nunca puede desaparecer completamente; [...] no hay camino de retorno en el desarrollo de la personalidad, sino sólo en la sucesión de las conductas [...]. Por más enfermo que esté un sujeto, este punto de coherencia no puede faltar» (Ibid., pp. 41 y 44) ${ }^{13}$.

13 Una idea muy similar se encuentra en la obra psiquiátrica de Luis Martín-Santos (1924-1964), quien también era de la opinión de que, en la medida en que la vida psíquica 
Y, por el otro, es incapaz de explicar por qué una determinada persona enferma psíquicamente en un momento dado y no en otro: «En la perspectiva evolucionista - concluye Foucault —, la enfermedad no tiene más ley que la de la virtualidad general» (Ibid., p. 45).

Por su parte, los importantes avances propiciados por el psicoanálisis habrían tenido, en opinión de Foucault, una relevancia todavía mayor para la historia de la psicología. A diferencia del pensamiento evolucionista, el psicoanálisis pudo apreciar desde un primer momento la dimensión biográfica del análisis psicopatológico, esto es, que

«el devenir psicológico es evolución e historia a la vez [y que] el tiempo del psiquismo debe analizarse según lo anterior y lo actual — es decir, en términos evolutivos - y también según lo pasado y lo presente - es decir, en términos históricos-. [...] El genio de Freud supo sobrepasar muy pronto este horizonte evolucionista [...] para llegar a la dimensión histórica del psiquismo» (Ibid., pp. 46-47).

En consecuencia, el psicoanálisis había promovido más que ninguna otra corriente el «descubrimiento del sentido», ya iniciado con la psicología de P. Janet o la psicopatología de K. Jaspers, pero que sólo Freud «llevó a sus límites extremos, dando con ello una nueva orientación a la psicología» (Foucault, 1994a, p. 157). De hecho, Freud fue tan lejos en la cuestión del sentido que acabó confiriéndole una objetividad que debía ser aprehendida a nivel de los símbolos contenidos en el «material» de la conducta - particularmente en el sueño como via regia hacia el inconsciente-, y, consecuentemente, siempre aspiró a convertir el psicoanálisis en una hermenéutica objetiva de psiquismo. Y justo aquí radicaron desde un principio las dificultades y divergencias del joven Foucault con el proyecto freudiano. Con su furor interpretativo y su noción inflacionaria del desciframiento del sentido, el psicoanálisis soslayaba dimensiones esenciales del psiquismo, $\mathrm{y}$, en su opinión, era víctima de una «teología de las significaciones [...] que agotan la realidad del mundo a través del cual ésta se anuncia» (Foucault, 1999, p. 70).

En su brillante prólogo a El sueño y la existencia, Foucault pudo concretar estas críticas en el marco de una extensa discusión de la concepción freudiana del sueño. El blanco de la crítica foucaultiana contenida en este texto es la tendencia del psicoanálisis a tratar el sueño como una simple referencia a factores externos - como traumas infantiles o pulsiones inconscientes-, así como su incapacidad para considerar la fuerza expresiva originaria e «innata» de las imágenes oníricas, que, en opinión de Foucault, antecede y supera cualquier análisis semántico: «el mundo imaginario tiene sus leyes propias, sus estructuras específicas; la imagen es algo más que el cumplimiento inme-

siempre remite a un cierto orden de sentido, los enfermos mentales nunca pierden totalmente la capacidad de autoorganización psíquica o psicomorfia y, por tanto, su capacidad de conformar una personalidad. Véase Martín-Santos (1955). 
diato del sentido; tiene su espesor» (Ibidem). Dicho en otros términos, el sueño habla un idioma que le es propio, y sus contenidos no pueden ser reducidos a meros deseos reprimidos, desplazados o enajenados ${ }^{14}$. Retomando la conocida terminología de F. de Saussure, Foucault concluye que «el psicoanálisis no dio al sueño otro estatuto sino el de la palabra [parole]; no supo reconocerlo en su realidad de lenguaje [langue]» (Ibidem). De forma análoga, el psicoanálisis había conseguido remitir el síntoma cardinal de la angustia a la historia psicológica del individuo, pero era incapaz de entrever el carácter fundamental y primigenio de la angustia como experiencia radicalmente original, esto es, «en su necesidad existencial» (Foucault, 1984, p. 62). En síntesis, pues, y a pesar de su reconocimiento explícito de la magnitud del legado freudiano, el joven Foucault tuvo desde un principio un abierto recelo al «imperialismo interpretativo» y al proceder hermenéutico del psicoanálisis, para el que, ciertamente, cada fenómeno psíquico constituye siempre una representación de algo que, en última instancia, nunca es él mismo.

La tercera y última corriente a considerar era la fenomenología, con la que Foucault, por formación y afinidad, se sentía indudablemente mucho más cómodo. Las insuficiencias del evolucionismo y el psicoanálisis tenían que ver, a su juicio, con el hecho de que tienden a entender los trastornos psíquicos como un proceso estrictamente natural o como la consecuencia de una historia, pero son incapaces de reconocer que estos trastornos no sólo son padecidos, sino también experimentados y vividos de forma activa, esto es, que implican la «constitución de un mundo». Por ese motivo, es necesaria una fenomenología de los trastornos mentales, una comprensión de la experiencia del enfermo «desde dentro», pues «sólo comprendiéndola desde el interior será posible ordenar en el universo mórbido las estructuras naturales constituidas por la evolución y los mecanismos individuales cristalizados por la historia psicológica» (Ibid., p. 63). Foucault demuestra conocer bien las aportaciones más relevantes de la fenomenología clínica, que agrupa según la distinción clásica entre análisis noético y noemático. En el caso del primero se trata, como es sabido, de rastrear la experiencia que el enfermo hace de su trastorno, mientras que el segundo intenta hacer inteligible el «universo patológico sobre el cual se abre esta conciencia de la enfermedad, el mundo que ella observa, y que al mismo tiempo la constituye» (Ibid., p. 66) ${ }^{15}$. Análoga-

14 Para Foucault, un ejemplo paradigmático de las limitaciones del proceder psicoanalítico con respecto al sueño lo ofrece el célebre «caso Dora» de Freud. En concreto, Foucault censura la insistencia freudiana en interpretar el contenido de los sueños de Dora como manifestaciones encubiertas de deseo sexual, hasta el punto de que Freud fue finalmente incapaz de reconocer su verdadero sentido como expresión de la decisión de Dora de asumir su soledad, emanciparse de su familia e incluso interrumpir el tratamiento con él. Cf. Foucault (1999), pp. 97-98, y Novella (2008), pp. 36-38.

15 Como aportaciones ejemplares a la fenomenología clínica, Foucault cita y muestra conocer con solvencia los trabajos de J. Wyrsch sobre la experiencia de la esquizofrenia y los estudios de E. Minkowski, E. Straus, L. Binswanger y R. Kuhn sobre las distorsiones en la cons- 
mente, la perspectiva fenomenológica y, más concretamente, la obra de Binswanger, posibilitan también una comprensión más completa del fenómeno del sueño, en la medida en que ven en él un lenguaje con entidad propia, una experiencia absolutamente original relacionada con el despliegue de la imaginación que sitúa al ser humano en contacto con lo trascendente, y cuya significación, por tanto, no puede limitarse a su posible análisis psicológico (Foucault, 1999).

En cualquier caso, y a pesar de su clara querencia por la fenomenología y el análisis existencial y la gran influencia de su lectura de Heidegger ${ }^{16}$, Foucault advirtió una serie de limitaciones en sus planteamientos que le llevaron a buscar otras alternativas. En una entrevista posterior, explicitó sus reservas en los siguientes términos:

«Por dos razones que no eran independientes entre sí [el análisis existencial] me dejaba insatisfecho: su insuficiencia teórica con respecto a la noción de experiencia y la ambigüedad de su vínculo con una práctica psiquiátrica que, al mismo tiempo, ignoraba y suponía» (Foucault 1994d, p. 579).

En su obra temprana, las insuficiencias de la fenomenología tenían que ver, más concretamente, con su tendencia a entender la experiencia y los trastornos mentales como fenómenos exclusivamente privados, olvidando así su constitución histórica y sus condiciones sociales y culturales de posibilidad. Foucault, en consecuencia, pensaba ya entonces que no era posible «dar cuenta de la experiencia patológica sin referirla a estructuras sociales, ni explicar las dimensiones psicológicas de la enfermedad [...] sin ver en el medio humano su condición real» (Foucault, 1984, p. 95). Dicho de otro modo, ni la evolución, ni la historia psicológica ni la reconstrucción fenomenológica de la experiencia mórbida podían explicar las condiciones de aparición de los trastornos mentales, pues «el hecho patológico tiene sus raíces en otra parte» (Ibid., p. 83) ${ }^{17}$.

titución de la temporalidad, espacialidad, corporalidad e intersubjetividad que caracterizan la experiencia mórbida (Foucault, 1984, pp. 66-77).

16 Sobre esta influencia ha insistido particularmente el filósofo norteamericano H. Dreyfus, que ha llegado a situar la ontología heideggeriana como un antecedente inmediato del concepto de poder elaborado por Foucault. Cf. Dreyfus (1996). Sin llegar a ese extremo, otros autores han propuesto más recientemente interpretaciones de la obra de Foucault que subrayan su profunda impronta fenomenológica. Cf. Ramos/Rejón (2002) o May (2005).

17 Para una presentación más detallada del tratamiento deparado a las diferentes corrientes psicopatológicas (y a sus respectivas insuficiencias) en la obra temprana de Foucault, véase Novella (2008). 


\section{Alienación y conflicto: La tentación de la sociogénesis}

En la segunda parte de Enfermedad mental y personalidad, Foucault ofreció un primer intento de análisis de estas condiciones históricas, sociales y culturales de la enfermedad mental. Aceptando la relatividad cultural de las leyes psicológicas y de lo "patológico» como un lugar común de la sociología o la antropología moderna, Foucault tenía además la convicción de que «en realidad, una sociedad se expresa positivamente en las enfermedades mentales que manifiestan sus miembros, cualquiera que sea el estatus que otorga a sus formas patológicas» (Ibid., p. 87). Con esta afirmación quería señalar que el fenómeno de la enfermedad mental, tal como se manifiesta en una determinada sociedad, posee un aspecto «positivo» o «real», esto es, que tanto sus contenidos concretos como el trato que se le dispensa son una expresión directa de esa misma sociedad. En consecuencia, el análisis «ecológico» de la enfermedad mental debe centrarse en el esclarecimiento de dos cuestiones básicas: por un lado, los requisitos históricos y culturales de la concepción y la «gestión» moderna de la enfermedad mental, y, por el otro, el modo y los medios concretos a través de los cuales la sociedad moderna se expresa en las «formas patológicas». Teniendo en cuenta su obra posterior, y con el objeto de apreciar mejor la importancia de su respuesta a la primera de estas cuestiones, abordaré en primer lugar su interpretación inicialmente marxista y sociogenética de la segunda.

En este punto, Foucault parte de la convicción de que los conflictos que los psiquiatras o los psicoanalistas creen ver en la biografías de sus pacientes se derivan, en realidad, de las experiencias contradictorias que atenazan la esfera social del capitalismo burgués: «Las relaciones sociales que determina la economía actual bajo las formas de la competencia, de la explotación, de guerras imperialistas y de luchas de clases ofrecen al hombre una experiencia de su medio humano acosada sin cesar por la contradicción» (Ibid., p. 98). En estas circunstancias, el análisis psicológico de la enfermedad mental -independientemente de que se opere desde el evolucionismo, el psicoanálisis o el análisis existencial - sólo puede resultar insuficiente, pues soslaya por completo este «conflicto real» en las condiciones de la existencia:

«las dimensiones psicológicas de la enfermedad no pueden ser encaradas como autónomas sin la ayuda de ciertos sofismas. Es verdad que podemos ubicar la enfermedad mental en relación a la evolución humana, en relación a las formas de existencia. Pero no debemos confundir estos diversos aspectos de la enfermedad con sus orígenes reales salvo que queramos recurrir a explicaciones míticas» (Ibid., p. 101) ${ }^{18}$.

18 Como un caso ejemplar de las distorsiones a que suele conducir una perspectiva exclusivamente psicológica, Foucault cita la introducción por parte de Freud del concepto de pulsión de muerte en Mas allá del principio del placer (1920), esto es, inmediatamente después de su traumática experiencia de la Primera Guerra Mundial: «Freud quería explicar la 
Sólo un análisis comprometido del medio social y de la alienación capitalista puede, por tanto, dar cuenta de la «verdadera» naturaleza y de la génesis de las enfermedades mentales.

Asumiendo esta posición en esos términos, Foucault se ve obligado a especificar los mecanismos concretos por medio de los cuales las contradicciones «reales» del medio social se transforman en conflictos psicológicos del individuo, es decir, el modo en que la alienación capitalista produce los trastornos psíquicos y se materializa la sociogénesis de la enfermedad mental. Con este fin, recurre a la conocida doctrina de los reflejos del fisiólogo ruso I. Pavlov, que, como es sabido, ofrece una explicación general del modo en que determinados estímulos externos generan ciertas respuestas del sistema nervioso ${ }^{19}$. Así, la aparición de la enfermedad mental radica en una «reacción de defensa difusa» o una «inhibición general» del sistema nervioso frente a una sobrecarga de estímulos generada por un entorno «mórbido»:

«en el momento en que las condiciones del medio ya no permiten la actividad normal del sistema nervioso, y que las contradicciones a las que está sometido el individuo ya no permiten la dialéctica normal de la excitación y de la inhibición, se instala una inhibición de defensa. Esta inhibición de defensa explica los mecanismos de la enfermedad» (Ibid., p. 112).

Normalmente, los individuos son capaces de afrontar los conflictos habituales en su medio social - por ejemplo, entre las necesidades de la vida familiar y las obligaciones laborales- por medio de reacciones «diferenciadas», esto es, por medio de «reacciones individualizadas a cada término o a cada fase de la situación conflictual» (Ibid., pp. 113-114). Cuando, por el contrario,

«el conflicto se presenta con un carácter de contradicción tan absoluta, o cuando las posibilidades del individuo están tan restringidas que la diferenciación no se puede efectuar, el individuo no puede defenderse más que colocándose fuera de circuito, respondiendo con una inhibición generalizada» (Ibid., p. 114).

Para Foucault, la enfermedad es pues un correlato de esta inhibición generalizada que ya no permite al individuo conducirse con soberanía en sus reacciones frente a las contradicciones del medio. En este sentido, es víctima de la alienación, pero no en el sentido clásico de una desviación o alejamiento genérico de la naturaleza humana, sino porque «ya no puede reconocerse en

guerra; pero es la guerra la que explica este giro del pensamiento freudiano» (Foucault, 1984, p. 99).

19 Para una exposición sumaria de los principios del pavlovismo y de su temprana aplicación a la comprensión de los trastornos mentales véase Pavlov (1967). Sobre su apropiación por parte de las élites estalinistas en el sentido de una doctrina marxista y soviética oficial de la fisiología humana, pueden consultarse los trabajos de Joravsky (1977) y Asratyan/Shingarov (1982). 
tanto que hombre en las condiciones de existencia que el hombre mismo ha instituido» (Ibidem).

Esta visión marxista representa, como se ha dicho, el primer intento foucaultiano de contemplar la enfermedad mental en su «necesidad» histórica, social y cultural, y debe verse indudablemente en el contexto de su temprana vinculación con los círculos comunistas del París de la época. La interpretación de Foucault es netamente sociogenética, en tanto explica la aparición de la enfermedad mental en un sentido estrictamente causal, atribuyendo su causa última a la configuración particularmente inhumana de la sociedad burguesa:

«[El enfermo mental] demuestra que la sociedad burguesa, por los mismos conflictos que han hecho posible su enfermedad, no está hecha a la medida del hombre real; que es abstracta en relación al hombre concreto y a sus condiciones de existencia; que continuamente pone en conflicto la idea unitaria que se hace del hombre y el status contradictorio que le otorga. El enfermo mental es la apoteosis de este conflicto. [...] La enfermedad es realmente la consecuencia de las contradicciones sociales en las que el hombre está históricamente alienado» (Ibid., p. 116).

En cierto modo, y como han señalado algunos autores, este enfoque explicativo parece encontrarse en una abierta contradicción con el espíritu fenomenológico que impregna su crítica a la psiquiatría positivista, la cual, como hemos visto, cuestionaba radicalmente las pretensiones explicativas de las ciencias naturales en el ámbito de la enfermedad mental en favor de una «descripción densa» de la experiencia vivida por el enfermo (May, 2005, pp. 298-299). Sin embargo, conviene recordar que semejante punto de vista estaba muy extendido en la Francia de la primera mitad de la década de 1950. Por un lado, aquellos años asistieron - como Foucault recordaría en una entrevista posterior - a los influyentes intentos de Sartre o Merleau-Ponty de conciliar la tradición fenomenológica con un compromiso político de signo marxista:

«No hay que olvidar que durante todo el período de 1945 a 1955 en Francia, toda la universidad francesa [...] estuvo muy preocupada y ocupada en construir algo que no era precisamente Freud-Marx, sino Husserl-Marx, el vínculo fenomenología-marxismo. Esta fue la apuesta de la contribución y los esfuerzos de toda una serie de gente; Merleau-Ponty, Sartre, yendo de la fenomenología al marxismo, se situaban en ese horizonte» (Foucault, 1994c, p. 434).

Y, por el otro, en los círculos marxistas de la época se sucedieron los intentos de elaborar una crítica radical de la psicología «burguesa» y del psicoanálisis ${ }^{20}$ y de esbozar los fundamentos de una psicopatología «materialis-

20 Bajo la influencia de G. Politzer, el psicoanálisis era visto en los cenáculos comunistas de la Francia de la época como un instrumento más de adaptación a la sociedad burguesa. Esta situación sólo cambiaría a partir de la década de 1960, cuando miembros prominentes del Par- 
ta» ${ }^{21}$. En cualquier caso, Foucault se distanció tempranamente de este entorno intelectual y, con ello, acabó abandonando el marco teórico ofrecido por el marxismo. Merced a esta desvinculación y al progresivo desarrollo de su propio pensamiento, su interpretación de las condiciones históricas y culturales de la enfermedad mental adoptaría pronto una forma muy distinta $y$, sin duda, mucho más interesante.

\section{Hacia una arqueología de la psicología y una ontología histórica de la enfermedad mental}

En 1962, un año después de la publicación de la Historia de la locura, Foucault presentó por expreso deseo de la editorial una segunda versión de Enfermedad mental y personalidad con el título Enfermedad mental y psicología (Foucault, 1962). Esta nueva versión se diferencia de la primera en tres aspectos decisivos que se corresponden, respectivamente, con tres elementos básicos de la nueva aproximación a la comprensión histórica de la enfermedad mental que Foucault desarrolla en la Historia de la locura ${ }^{22}$.

La diferencia más evidente es, sin duda, el distanciamiento de los principios y categorías marxistas, que se hace especialmente patente en la supresión de todo el intento de utilizar la fisiología de los reflejos de Pavlov como una suerte de fundamento materialista para la transformación de contradicciones sociales en conflictos psicológicos. También en la descripción de las condiciones sociales de la enfermedad mental, Foucault renuncia en gran medida a su recurso anterior a la terminología marxista. En lugares donde en 1954 había hablado de «capitalismo», «imperialismo» o de «determinaciones económicas de las contradicciones sociales», en la nueva edición remite simplemente a «nuestra cultura» o a la «experiencia que hace nuestra cultura de sus propias contradicciones» (Foucault, 1962, pp. 125-126). Esto no implica que abandone la utilización de cualquier concepto de inspiración marxista — de hecho, todavía alude en algunos pasajes a la «explotación» o a la «lucha

tido Comunista como L. Althusser empezaron a desarrollar, bajo la influencia de J. Lacan, una nueva actitud frente al legado freudiano. Véase a este respecto Turkle, 1980, pp. 217-224.

${ }^{21}$ En 1951, por ejemplo, el psicólogo evolutivo H. Wallon fundó la revista La Raison: Cahiers de psychopathologie scientifique, en la que se glosaron con grandes elogios los trabajos de Pavlov y los logros de la fisiología soviética. También la publicación comunista La Nouvelle Critique - en la que Foucault colaboró puntualmente - se ocupó a menudo durante esos años de los problemas de la psicología y la psiquiatría desde este punto de vista materialista. Véase Eribon (1992), pp. 84-105.

22 Por ese motivo, y como han señalado Macherey (1986), pp. 752-754, Gutting (1989), p. 69, o May (2005), pp. 300-301, esta segunda edición constituye un texto extremadamente interesante para advertir la evolución y los desplazamientos más significativos experimentados por el pensamiento de Foucault en el período de preparación y redacción de la Historia de la locura. 
de clases» (Ibid., p. 125) - , pero ésta se da sólo de forma ocasional y no impregna en el mismo grado el contenido y el espíritu del texto ${ }^{23}$. En cualquier caso, el distanciamiento del marxismo se muestra, ante todo, en su abandono definitivo de dos ideas clave que habían tenido un papel muy relevante en todo su planteamiento anterior. En primer lugar, Foucault sustituye la perspectiva de una sociogénesis en el sentido causal de una producción social de la enfermedad mental por la noción algo más compleja de una «constitución» 0 «surgimiento» de la enfermedad mental en un determinado contexto histórico y cultural; en este caso, el objetivo ya no consiste en la identificación de una causa última para el fenómeno de la enfermedad mental - cuya «realidad», por lo demás, no resulta cuestionada-, sino en la clarificación de su «contexto de constitución», esto es, en la reconstrucción histórica de los eventos concretos que han hecho posible nuestra concepción y nuestra percepción de la enfermedad mental como tal. Y, en segundo lugar, el joven filósofo abandona la noción hegeliano/marxista de un «hombre real» $\mathrm{u}$ «hombre mismo» no alienado y perfectamente racional como punto de referencia de su crítica; consecuentemente, ya no entiende la enfermedad mental - a la que se refiere ahora de forma intencionadamente difusa como «locura» o «sinrazón»- como el resultado de la alienación social de un hombre postulado en términos idealistas, sino, simplemente, como una posibilidad fáctica de la humanidad que sólo con la mirada de la psicología moderna se constituye en enfermedad mental.

Significativamente, la segunda diferencia esencial entre las dos ediciones consiste en un desplazamiento desde la focalización en la enfermedad mental como problema psicológico o psicopatológico hacia el problemático estatus de la psicología misma. Este desplazamiento se anuncia en el nuevo título, pero se hace más evidente en la nueva perspectiva foucaultiana con respecto a las condiciones sociales y culturales de la enfermedad mental. Mientras el enfoque de la primera edición implicaba — como ya se ha apuntado - una asunción implícita de la categoría de la enfermedad mental, Foucault parte en la versión posterior de la convicción de que, en realidad, esta categoría es una singular «invención» de la cultura occidental que sólo puede entenderse en relación con el nacimiento de la psicología y la psiquiatría moderna en el

${ }^{23}$ Hay que suscribir las palabras de G. Gutting cuando dice que «Foucault parece mantener gran parte de la insatisfacción marxista con la sociedad burguesa, pero está menos convencido de los análisis y los remedios marxistas ortodoxos para afrontar sus deficiencias» (Gutting, 1989, pp. 66-67). En cualquier caso, la influencia del marxismo estará presente en muchos aspectos de su obra posterior, y, especialmente, en la visión de la relación entre teoría y praxis que inspira su análisis de diversas prácticas — psicológicas, penitenciarias, sexuales o de subjetivación - y, en general, todo su enfoque genealógico. Igual que Marx, Foucault mantendrá siempre la convicción de que las prácticas sociales son productos contingentes de un determinado desarrollo histórico, y de que todo saber se encuentra estrechamente vinculado a las relaciones de poder existentes en el seno de una determinada sociedad. Véase, en este sentido, Smart (1983), Poster (1984), McDonald (2002) y Olssen (2004). 
tránsito de los siglos XVIII al XIX. La enfermedad mental ya no es pues el producto de un determinado orden social, sino una determinada forma de ver la locura dentro de una cultura que le ha sustraído cualquier objetividad más allá de la afectación o discapacidad del psiquismo. Y, en consecuencia, la aportaciones de la psicología y la psiquiatría moderna no han de verse como «un descubrimiento progresivo de aquello que la locura es en la verdad de su ser, sino [como] el sedimento de lo que la historia de Occidente ha hecho con ella desde hace trescientos años» (Ibid., p. 107). En pocas palabras: la fabricación social de la locura se ha convertido en una arqueología de la psicología ${ }^{24}$.

Finalmente, el texto de 1962 se distingue por la presencia de una idea decisiva que atraviesa todo el planteamiento de la Historia de la locura, y que puede definirse como la «historización de las formas de experiencia». Foucault señala, en este sentido, que los problemas epistemológicos que conlleva el hecho de tomar las dimensiones psicológicas de la enfermedad como «datos absolutos» pueden evitarse si los distintos aspectos de la enfermedad se conciben como «formas ontológicas» susceptibles de ser examinadas en su constitución histórica: «en realidad, sólo en la historia puede encontrarse el a priori concreto a partir del cual la enfermedad mental toma sus figuras necesarias con la apertura vacía de su posibilidad» (Ibid., p. 129). Con ello, el joven filósofo ponía en pie un proyecto teórico que, en cierto modo, constituye una especie de aplicación histórica del análisis existencial, esto es, una ampliación de la perspectiva fenomenológica con el objetivo expreso de captar la constitución del mundo de la experiencia de épocas pretéritas y de descubrir en él las condiciones estructurales de aparición de determinados fenómenos (Flynn, 1997). Estas raíces fenomenológicas del proyecto de una «ontología histórica» - que la Historia de la locura acometería de forma específica con respecto a la enfermedad mental-, pareció confirmarlos el propio Foucault en un texto posterior: «estudiar así, en su historia, las formas de experiencia es un tema que surgió de un proyecto más antiguo: el de hacer uso de los métodos del análisis existencial en el campo de la psiquiatría y en el dominio de la enfermedad mental» (Foucault, 1994d, p. 579).

En síntesis, puede decirse que, en el camino hacia la Historia de la locu$r a$, Foucault esbozó una nueva aproximación a los problemas de la psicología y la enfermedad mental que se caracterizaba por el distanciamiento del marxismo $-\mathrm{y}$, con él, de la perspectiva de la sociogénesis y del idealismo del «hombre real»-, la problematización arqueológica de la psicología y la historización de la experiencia de la locura. Sin embargo, en este punto conviene subrayar que una lectura atenta de los escritos de la década de 1950 permite identificar con claridad toda una serie de anticipaciones de estas ideas, de ma-

24 Como es sabido, esta idea encontrará su célebre formulación en la Historia de la locura: «Haciendo la historia del loco, hemos hecho - [...] siguiendo el encadenamiento de las estructuras fundamentales de la experiencia - la historia de aquello que ha hecho posible la aparición misma de una psicología» (Foucault, 1972, p. 548). 
nera que es legítimo pensar que, en una porción muy considerable, todas ellas proceden de intuiciones muy tempranas de Foucault que, al cabo de un tiempo, cristalizarían en los conocidos planteamientos de la Historia de la locura.

En relación con el distanciamiento del marxismo, es sabido, por ejemplo, que éste se inició de una forma relativamente temprana. Foucault, de hecho, abandonó el Partido Comunista de Francia (PCF) en 1953 - no está claro si como consecuencia de las mentiras estalinistas con respecto al «complot de los médicos» de 1952 o, como L. Althusser sugeriría más tarde, a causa de su homosexualidad (Eribon, 1992, pp. 88-89) —. No obstante, ni siquiera en su período de militancia fue un marxista dogmático, como prueban con claridad su interés en la psicología evolutiva de J. Piaget o la fenomenología, sus tempranas lecturas de Heidegger o Nietzsche o sus propios escritos del período.

Por su parte, también la problematización arqueológica de la psicología se anuncia en los primeros textos del joven Foucault, particularmente en los dos ensayos de 1957 «La psicología desde 1850 a 1950» y «La investigación científica y la psicología». De hecho, Foucault ofrece en estos textos una aguda crítica de la psicología positivista que ya contiene algunos elementos clave de su posterior «ajuste de cuentas»: el origen histórico de la psicología «científica» a partir de determinadas «contradicciones» con las que el ser humano se encuentra en su praxis; la intención normalizadora — más tarde dirá moralizadora - que subyace a su presunta orientación a los «hechos»; o el forzoso olvido de su papel dentro de las dimensiones de la negatividad del hombre y de su «vocación eternamente infernal» (Foucault, 1994b, pp. 186). En síntesis, puede darse por seguro que Foucault había reconocido muy pronto numerosos problemas en el estatuto epistemológico y en la proyección cultural de la psicología, y que ya entonces le había prescrito un «retorno a los infiernos» con el fin de que el hombre - como escribiría poco después - «pueda ser algún día libre para el gran encuentro trágico con la locura» (Foucault, 1962, p. 115).

Por último, la idea de que los problemas de la psicología «tienen que ver más con razones históricas profundas que con un simple desplazamiento cultural» (Foucault, 1994b, pp. 178) se encuentra entre las convicciones más precoces del joven Foucault. Ya en la primera edición de Enfermedad mental $y$ personalidad ofreció, en un capítulo titulado «El sentido histórico de la alienación mental», una interesante síntesis de las condiciones históricas de la concepción moderna y la gestión manicomial de la enfermedad mental (Foucault, 1984, pp. 88-102). Ciertamente, en este capítulo no intentó todavía reconstruir la experiencia de la locura en distintos períodos históricos ni vincular el nacimiento de la psicología y la psiquiatría «científica» con una determinada relación cultural con la locura - «definida externamente por la exclusión y el castigo, e internamente por la moralización y la culpa» (Foucault, 1962, p. 113), como describiría poco después. En cualquier caso, ya entonces señalaba que «en realidad, sólo en la historia se pueden descubrir las 
condiciones de posibilidad de las estructuras psicológicas» (Foucault, 1984, p. 102), anticipando algunos análisis que no sólo no encajaban con la tesis de una producción social de los fenómenos psicopatológicos, sino que se aproximaban notablemente a la perspectiva posterior de una constitución histórica de los mismos 25 .

En conclusión, todas estas consideraciones permiten apreciar la complejidad del largo camino que requirió la génesis de la Historia de la locura, un camino en el que Foucault transitó con gran autoridad los territorios de la fenomenología y - si bien con menor convicción - del marxismo y pudo dotarse de algunas herramientas teóricas decisivas que marcarían su devenir filosófico. Con todo, lo expuesto también muestra con claridad que el despliegue posterior de su pensamiento se basa en numerosas ideas e intuiciones que se remontan al período más temprano de su producción. En realidad, era sólo una cuestión de tiempo - del tiempo que pasó en el «largo invierno sueco» de la segunda mitad de la década de 1950 - que consiguiera articularlas en un enfoque más «maduro», personal y consistente.

\section{BIBLIOGRAFÍA}

Asratyan, E. A. y Shingarov, G. K. (1982): «Lenin's theory of reflection and Pavlov's teaching on higher nervous activity», Neuroscience and Behavioral Physiology, 12: 357-363.

BARTHES, R. (1961): «Savoir et folie», Critique, 17: 915-922.

Blasius, D. (1986): «Psychiatriereform und Psychiatriegeschichte», en Umgang mit Unheilbaren: Studien zur Sozialgeschichte der Psychiatrie, Psychiatrie-Verlag, Bonn, pp. 81-89.

CAnguilhem, G. (1968): «Qu'est-ce que la psychologie?» en Études d'histoire et de philosophie des sciences, Vrin, París, pp. 365-381.

CASTEL, R. (1980): El orden psiquiátrico: La edad de oro del alienismo, La Piqueta, Madrid.

- (1994): «Problematization as a mode of reading history», en: Goldstein, J. (Ed.): Foucault and the Writing of History. Blackwell, Oxford, pp. 237-252.

CheBILI, S. (2005): Foucault et la psychologie, L'Harmattan, París.

Cranston, M. (1973): «Michel Foucault: A structuralist view of reason and madness», en The Mask of Politics and Other Essays, Liberty Press, Nueva York, pp. $137-155$.

Deleuze, G. (1986): Foucault. Editions de Minuit, París (Español: Paidós, Barcelona, 1987).

25 Como un ejemplo de estos análisis pueden tomarse sus comentarios con respecto a la laicización de la cultura como requisito para la aparición de delirios religiosos: «La religión puede ser objeto de una creencia delirante en la medida en que la cultura de un grupo no permite asimilar las creencias religiosas al contenido actual de la experiencia» (Foucault, 1984, p. 97). 
DerridA, J. (1964): «Cogito et histoire de la folie», Revue de Metaphysique et de Morale, 3/4: 460-494.

DöRnER, K. (1969): Bürger und Irre: Zur Sozialgeschichte und Wissenschaftssoziologie der Psychiatrie, Europäische Verlagsanstalt, Francfort (Español: Taurus, Madrid, 1974).

Dreyfus, H. (1987): «Foucault's critique of psychiatric medicine», The Journal of Medicine and Philosophy, 12: 311-333.

- (1996): «Being and power: Heidegger and Foucault», International Journal of Philosophical Studies, 4: 1-16.

Engstrom, E. J.; Weber, M. M. y Hoff, P. (Ed.) (1999): Knowledge and Power: Perspectives in the history of psychiatry, Verlag Wissenschaft und Bildung, Múnich.

ERIBON, D. (1992): Michel Foucault (1926-1984), Anagrama, Barcelona.

Évolution psychiatrique (1971): «La conception idéologique de l'Histoire de la Folie de Michel Foucault (Journées annuelles de l'Evolution Psychiatrique, Toulouse, 6-7 Décembre 1969)», Évolution psychiatrique 36: 221-298.

FlaherTy, P. (1986): «(Con)textual contest: Derrida and Foucault on madness and the Cartesian subject», Philosophy of Social Science 16: 157-175.

FLYNN, T. R. (1997): Sartre, Foucault and Historical Reason, Volumen I: Toward an existentialist theory of history, Chicago University Press, Chicago.

- (2005): «Foucault's mapping of history», en GutTing, G. (Ed.), The Cambridge Companion to Foucault, 2. ${ }^{\mathrm{a}}$ ed., Cambridge University Press, Nueva York, pp. 29-48.

Foucault, M. (1962): Maladie mentale et psychologie, Presses Universitaires de France, París.

- (1972): Histoire de la folie à l'âge classique, 2. a ed., Gallimard, París (Español: FCE, México, 1976).

— (1983): « Afterword: The subject and power», en Dreyfus, H. y Rabinow, P., Michel Foucault: Beyond structuralism and hermeneutics, 2. ${ }^{\mathrm{a}}$ ed., Chicago University Press, Chicago, pp. 214-232.

- (1984): Enfermedad mental y personalidad. Paidós, Barcelona.

- (1994a): «La psychologie de 1850 à 1950», en Dits et écrits, Volumen I, Gallimard, París, pp. 148-165 (Español: Archipiélago 34/35: 163-173, 1998).

- (1994b): «La recherche scientifique et la psychologie», en Dits et écrits, Volumen I. Gallimard, París, pp. 165-186.

— (1994c): «Structuralisme et poststructuralisme (Entretien avec G. Raulet, 1983)», en Dits et écrits, Volumen IV, Gallimard, París, pp. 431-457.

- (1994d): «Préface à l'Histoire de la sexualité», en Dits et écrits, Volumen IV, Gallimard, París, pp. 578-584.

- (1994e): «Qu'est-ce que les Lumières?», en Dits et écrits, Volumen IV. Gallimard, París, pp. 679-688.

- (1999): «Introducción», en Entre filosofía y literatura: Obras esenciales I, Paidós, Barcelona, pp. 65-120.

Goldstein, J. (Ed.) (1994): Foucault and the Writing of History, Blackwell, Oxford.

Gros, F. (1997): Foucault et la folie, Presses Universitaires de France, París.

Gutting, G. (1989): Michel Foucault's Archaeology of Scientific Reason, Cambridge University Press, Cambridge. 
- (1994): «Michel Foucault's Phänomenologie des Krankengeistes», en Micale, M. S. y Porter, R. (Ed.), Discovering the History of Psychiatry, Oxford University Press, Nueva York, pp. 331-347.

- (2005): «Foucault and the history of madness», en The Cambridge Companion to Foucault, 2. ${ }^{\mathrm{a}}$ ed., Cambridge University Press, Nueva York, pp. 49-73.

History of the Human Sciences (1990): «Histoire de la folie: An unknown book by Michel Foucault», History of the Human Sciences, 3: 1-67.

Huertas, R. (2006): «Foucault, treinta años después. A propósito de El poder psiquiátrico», Asclepio, 58(2): 267-276.

Jones, C. y Porter, R. (Ed.) (1994): Reassessing Foucault: Power, medicine and the body, Routledge, Londres.

JoRAVSKY, D. (1977): «The mechanical spirit: The stalinist marriage of Pavlov to Marx», Theory and Society, 4: 457-477.

Labreure, D. (2004): Michel Foucault: Psychiatrie et médecine, Université Paris I Sorbonne, París.

Lloyd, M. y Thacker, A. (Ed.) (1997): The Impact of Michel Foucault on the Social Sciences and Humanities, St. Martin's Press, Nueva York.

MAcherey, P. (1986): «Aux sources de l'Histoire de la folie: une rectification et ses limits», Critique, 43: 752-774.

Macey, D. (1994): The Lives of Michel Foucault, Pantheon Books, Nueva York (Español: Cátedra, Madrid, 1995).

MANDrou, R. (1962): «Trois clefs pour comprendre la folie à l'époque classique», Annales ESC, 4: 761-772.

MARTín-SAnTOS, L. (1955): «Fundamentos teóricos del conocer psiquiátrico», Theoria, 3: 53-66.

MAY, T. (2005): «Foucault's relation to phenomenology», en GuTTING, G. (Ed.), The Cambridge Companion to Foucault, 2. ${ }^{\mathrm{a}}$ ed., Cambridge University Press, Nueva York, pp. 284-311.

McDonald, B. J. (2002): «Marx, Foucault, genealogy», Polity, 34: 259-275.

Megill, A. (1987): «The reception of Foucault by historians», Journal of the History of the Ideas, 48: 117-141.

Midelfort, H. C. E. (1980): «Madness and civilization in early modern Europe: A reappraisal of Michel Foucault», en Malament, B. (Ed.), After the Reformation: Essays in honour of J.H. Hexter, University of Pennsylvania Press, Filadelfia, pp. 247-265.

MiLler, J. (1993): The passion of Michel Foucault, Harper\&Collins, Londres (Español: Editorial Andrés Bello, Santiago de Chile, 1996).

Moreno Pestaña, J. L. (2006): Convirtiéndose en Foucault: Sociogénesis de un filósofo, Montesinos, Barcelona.

Novella, E. J. (2008): Der junge Foucault und die Psychopathologie: Psychiatrie und Psychologie im frühen Werk von Michel Foucault, Logos Verlag, Berlín.

Olssen, M. (2004): «Foucault and marxism: Rewriting the theory of historical materialism», Policy Futures in Education 2: 454-482.

PAVlov, I. P. (1967): Psicopatología y psiquiatría, psicofisiología experimental, reflexología y tipología: Problemas y aplicaciones, Morata, Madrid.

Poster, M. (1982): «Foucault and history», Sociological Research, 49: 116-142.

- (1984): Foucault, Marxism, and History, Polity Press, Cambridge. 
RAMOs, P. y REJón, C. (2002): El esquema de lo concreto: Una introducción a la psicopatología, Triacastela, Madrid.

Rothman, D. (1971): The Discovery of the Asylum: Social order and disorder in the new republic, Little Brown, Boston MA.

Roudinesco, E. (Ed.) (1992): Penser la folie: Essais sur Michel Foucault, Editions Galilée, París.

Russ, J. (1979): Histoire de la folie: Michel Foucault, Hatier, París.

SARASIN, P. (2005): Foucault zur Einführung, Junius Verlag, Hamburgo.

SCUll, A. (1979): Museums of Madness: The social organization of insanity in nineteenth-century England, Allen Lane, Londres.

Sedgwick, P. (1981): «Michel Foucault: The anti-history of psychiatry», Psychological Medicine, 11: 235-248.

Smart, B. (1983): Foucault, Marxism, and Critique, Routledge\&Kegan Paul, Londres.

Still, A. y Velody, I. (Ed.) (1992): Rewriting the History of Madness: Studies in Foucault's "Histoire de la folie", Routledge, Londres.

Stone, L. (1982): «Madness», New York Review of Books, 29(20): 28-36.

Turkle, S. (1980): «La antipsiquiatría francesa», en InglebY, D. (Ed.), Psiquiatría crítica: La política de la salud mental, Crítica, Barcelona, pp. 193-235.

VeYne, P. (1992): Foucault revoluciona la historia, Alianza, Madrid.

Von WeIZSÄCKer, V. (1958) Le cycle de la structure [Der Gestaltkreis], Desclée de Brouwer, París. 